\title{
Unsteady Stagnation-point Flow of a Second-grade Fluid
}

\author{
Fotini Labropulu ${ }^{1}$, Daiming $\mathbf{L i}^{2}$ \\ ${ }^{1}$ Luther College/University of Regina \\ Regina, SK, Canada S4S 0A2 \\ fotini.labropulu@uregina.ca \\ 2Department of Chemical and Petroleum Engineering, University of Calgary \\ 2500 University Dr. NW, Calgary, Alberta, Canada T2N 1N4
}

\begin{abstract}
The unsteady two-dimensional stagnation point flow of second-grade fluid impinging on an infinite plate is examined and solutions are obtained. It is assumed that the infinite plate at $y=0$ is oscillating with velocity $U \cos \Omega t$, the fluid occupies the entire upper half plane $y>0$ and it impinges obliquely on the plate. The governing partial differential equations are reduced to a system of ordinary differential equations by assuming a form of the streamfunction a priori. The resulting equations are, then, solved numerically using a shooting method for various values of the Weissenberg number, We. It is observed that the effect of the Weissenberg number is to decrease the velocity near the wall as it increases. Furthermore, analytical solutions are obtained for small and large values of frequency.
\end{abstract}

Keywords: Unsteady, Stagnation-point, Oscillating plate, Non-Newtonian fluid.

(C) Copyright 2016 Authors - This is an Open Access article published under the Creative Commons Attribution License terms (http://creativecommons.org/licenses/by/3.0). Unrestricted use, distribution, and reproduction in any medium are permitted, provided the original work is properly cited.

\section{Nomenclature}

$\begin{array}{ll}A_{1}, A_{2} & 1^{\text {st }} \text { and } 2 \text { nd Rivlin Ericksen tensors } \\ F(y), G(y, t) & \text { similarity variables } \\ k & \text { constant } \\ p & \text { fluid pressure } \\ T & \text { Cauchy Stress Tensor } \\ V & \text { velocity vector } \\ & \text { velocity components along } x \text { and } y \\ u, v & \text { axis } \\ U & \text { constant } \\ W e & \text { Weissenberg number }\end{array}$

Date Received: 2015-09-04

Date Accepted: 2016-01-26

Date Published:

\author{
Greek Symbols \\ $\alpha_{1}, \alpha_{2}$ \\ $\beta=\frac{\Omega}{k}$ \\ $\gamma \quad$ constant \\ $\varepsilon=\frac{U}{\sqrt{v k}} \quad$ constant \\ $\eta$ \\ $\mu$ \\ $\nu$ \\ $\tau_{12}$ \\ $\tau=\Omega t$ \\ $\psi$ \\ $\Omega$
}

\section{Introduction}

In the past, the fluid flow near a stagnation point has been investigated extensively. Hiemenz [1] derived an exact solution of the steady flow of a Newtonian fluid impinging orthogonally on an infinite flat plate. Stuart [2], Tamada [3] and Dorrepaal [4] independently investigated the solutions of a stagnation point flow when the fluid impinges obliquely on the plate. Beard and Walters [5] used boundary-layer equations to study two-dimensional flow near a stagnation point of a viscoelastic fluid. Dorrepaal et al [6] investigated the behaviour of a viscoelastic fluid impinging on a flat rigid wall at an arbitrary angle of incidence. Labropulu et al [7] studied the oblique flow of a viscoelastic fluid impinging on a porous wall with suction or blowing.

Unsteady stagnation point flow of a Newtonian fluid has also been studied extensively. Rott [8] and Glauert [9] studied the stagnation point flow of a Newtonian fluid when the plate performs harmonic oscillations in its own plane. Srivastava [10] 
investigated the same problem for a non-Newtonian second grade fluid using the Karman-Pohlhausen method [11] to solve the resulting equations. Labropulu et al [12] used series methods to solve the unsteady stagnation point flow of a Walters' B' fluid impinging on an oscillating flat plate. Matunobu $[13,14]$ and Kawaguti and Hamano [15] examined the fundamental character of the unsteady flow near a stagnation point for a Newtonian fluid. Takemitsu and Matunobu [16] studied the oblique stagnation point flow for a Newtonian fluid and obtained the general features of a periodic stagnation point flow. The case when the stagnation point fluctuates along a solid boundary is especially interesting from the biomechanical point of view. This is because the wall shear stress experienced by blood vessels may be thought to be increased by pulsating blood flow near the mean position of fluctuating stagnation point $[15,17]$ and lead to vascular diseases [18].

In this work, the unsteady stagnation point flow of a viscoelastic second-grade fluid is examined and solutions are obtained. We assume that the infinite plate at $y=0$ is oscillating with velocity $U \cos \Omega t$, the fluid occupies the entire upper half plane $y>0$ and the fluid impinges obliquely on the plate. The governing partial differential equations are reduced to a system of ordinary differential equations by assuming a form of the streamfunction a priori. The resulting equations are, then, solved numerically using a shooting method for various values of the Weissenberg number, $W e$. It is observed that the effect of the Weissenberg number is to decrease the velocity near the wall as it increases. Furthermore, analytical solutions are obtained for small and large values of frequency.

\section{Flow Equations}

The flow of a viscous incompressible nonNewtonian second-grade fluid, neglecting thermal effects and body forces, is governed by

$\operatorname{div} V=0$

$\rho \dot{V}=\operatorname{div} T$

when the constitutive equation for the Cauchy stress tensor $T$ which describes second-grade fluid given by Rivlin and Ericksen [19] is

$$
\left.\begin{array}{c}
T=-p I+\mu A_{1}+\alpha_{1} A_{2}+\alpha_{2} A_{1}^{2} \\
A_{1}=(\operatorname{grad} V)+(\operatorname{grad} V)^{T} \\
A_{2}=\dot{A}_{1}+(\operatorname{grad} V)^{T} A_{1}+A_{1}(\operatorname{grad} V)
\end{array}\right\}
$$

Here $V$ is the velocity vector field, $p$ the fluid pressure function, $\rho$ the constant fluid density, $\mu$ the constant coefficient of viscosity and $\alpha_{1}, \alpha_{2}$ the normal stress moduli. Dunn and Fosdick [20] and Dunn and Rajagopal [21] have shown that if the second-grade fluid described by (3) is to undergo motions which are compatible with Clausius-Duhem inequality [22] and the assumption that the free energy density of the fluid be locally at rest, then the material constants must satisfy the following restrictions:

$\mu \geq 0, \quad \alpha_{1} \geq 0, \quad \alpha_{1}+a_{2}=0$

Considering the flow to be plane, we take $V=(u(x, y, t), v(x, y, t))$ and $p=p(x, y, t)$ so that the flow equations (1) to (3) take the form

$$
\begin{aligned}
& \frac{\partial u}{\partial x}+\frac{\partial v}{\partial y}=0 \\
& \frac{\partial u}{\partial t}+u \frac{\partial u}{\partial x}+v \frac{\partial u}{\partial y}+\frac{1}{\rho} \frac{\partial p}{\partial x}=v \nabla^{2} u+\frac{\alpha_{1}}{\rho}\left\{\frac{\partial}{\partial t}\left(\nabla^{2} u\right)\right. \\
& +\frac{\partial}{\partial x}\left[2 u \frac{\partial^{2} u}{\partial x^{2}}+2 v \frac{\partial^{2} u}{\partial x \partial y}+4\left(\frac{\partial u}{\partial x}\right)^{2}+2 \frac{\partial v}{\partial x}\left(\frac{\partial v}{\partial x}+\frac{\partial u}{\partial y}\right)\right] \\
& +\frac{\partial}{\partial y}\left[\left(u \frac{\partial}{\partial x}+v \frac{\partial}{\partial y}\right)\left(\frac{\partial v}{\partial x}+\frac{\partial u}{\partial y}\right)+2 \frac{\partial u}{\partial x} \frac{\partial u}{\partial y}\right. \\
& \left.\left.+2 \frac{\partial v}{\partial x} \frac{\partial v}{\partial y}\right]\right\}+\frac{\alpha_{2}}{\rho} \frac{\partial}{\partial x}\left[\left(\frac{\partial u}{\partial x}\right)^{2}+\left(\frac{\partial v}{\partial x}+\frac{\partial u}{\partial y}\right)^{2}\right]
\end{aligned}
$$

$$
\begin{aligned}
& \frac{\partial v}{\partial t}+u \frac{\partial v}{\partial x}+v \frac{\partial v}{\partial y}+\frac{1}{\rho} \frac{\partial p}{\partial y}=v \nabla^{2} v+\frac{\alpha_{1}}{\rho}\left\{\frac{\partial}{\partial t}\left(\nabla^{2} v\right)\right. \\
& +\frac{\partial}{\partial x}\left[\left(u \frac{\partial}{\partial x}+v \frac{\partial}{\partial y}\right)\left(\frac{\partial v}{\partial x}+\frac{\partial u}{\partial y}\right)+2 \frac{\partial u}{\partial x} \frac{\partial u}{\partial y}+2 \frac{\partial v}{\partial x} \frac{\partial v}{\partial y}\right] \\
& +\frac{\partial}{\partial y}\left[2 u \frac{\partial^{2} v}{\partial x \partial y}+4\left(\frac{\partial v}{\partial y}\right)^{2}+2 \frac{\partial u}{\partial y}\left(\frac{\partial v}{\partial x}+\frac{\partial u}{\partial y}\right)\right. \\
& \left.\left.+2 v \frac{\partial^{2} v}{\partial y^{2}}\right]\right\}+\frac{\alpha_{2}}{\rho} \frac{\partial}{\partial y}\left[\left(\frac{\partial v}{\partial y}\right)^{2}+\left(\frac{\partial v}{\partial x}+\frac{\partial u}{\partial y}\right)^{2}\right]
\end{aligned}
$$


where $v=\frac{\mu}{\rho}$ is the kinematic viscosity.

Continuity equation (5) implies the existence of a streamfunction $\psi(x, y, t)$ such that

$$
u=\frac{\partial \psi}{\partial y}, \quad v=-\frac{\partial \psi}{\partial x}
$$

Substitution of (8) in equations (6) and (7) and elimination of pressure from the resulting equations using $p_{x y}=p_{y x}$ yields

$$
\begin{aligned}
\frac{\partial}{\partial t}\left(\nabla^{2} \psi\right)-\frac{\alpha_{1}}{\rho} \frac{\partial}{\partial t} & \left(\nabla^{4} \psi\right)-\frac{\partial\left(\psi, \nabla^{2} \psi\right)}{\partial(x, y)}+\frac{\alpha_{1}}{\rho} \frac{\partial\left(\psi, \nabla^{4} \psi\right)}{\partial(x, y)} \\
-v \nabla^{4} \psi & =0
\end{aligned}
$$

Having obtained a solution of equation (9), the velocity components are given by (8) and the pressure can be found by integrating equations (6) and (7).

The shear stress component $\tau_{12}$ of the Cauchy stress $T$ is given by

$$
\begin{aligned}
\tau_{12} & =\mu\left[\frac{\partial^{2} \psi}{\partial y^{2}}-\frac{\partial^{2} \psi}{\partial x^{2}}\right]+\alpha_{1}\left[\frac{\partial \psi}{\partial y}\left(\frac{\partial^{3} \psi}{\partial x \partial y^{2}}-\frac{\partial^{3} \psi}{\partial x^{3}}\right)\right. \\
- & \left.\frac{\partial \psi}{\partial x}\left(\frac{\partial^{3} \psi}{\partial y^{3}}-\frac{\partial^{3} \psi}{\partial x^{2} \partial y}\right)+2 \frac{\partial^{2} \psi}{\partial x \partial y} \frac{\partial^{2} \psi}{\partial y^{2}}+2 \frac{\partial^{2} \psi}{\partial x^{2}} \frac{\partial^{2} \psi}{\partial x \partial y}\right]
\end{aligned}
$$

\section{Solutions in the Fixed Frame of Reference}

Following Takemitsu and Matunobu [16], we assume that

$$
\psi=k[x f(y)+g(y, t)]
$$

We assume that the infinite plate at $y=0$ is oscillating with velocity $U \cos \Omega t$ and that the fluid occupies the entire upper half plane $y>0$. Furthermore, we assume the streamfunction far from the wall is given by $\psi=\frac{1}{2} \gamma y^{2}+x y$ (see Stewart [2]). Thus, the boundary conditions are given by

$$
\begin{aligned}
& f(0)=f^{\prime}(0)=0, \quad g(0, t)=0, \quad g_{y}(0, t)=\frac{U}{k} e^{i \Omega t} \\
& f^{\prime}(\infty)=1, \quad g_{y}(\infty, t)=\gamma y
\end{aligned}
$$

where $\gamma$ is a non-dimensional constant characterizing the obliqueness of oncoming flow. It is assumed that only the real part of a complex quantity has its physical meaning.

Substitution of equation (11) in (9) yields

$$
v f^{(i v)}+k\left(f f^{\prime \prime \prime}-f^{\prime} f^{\prime \prime}\right)-\frac{\alpha_{1} k}{\rho}\left(f f^{(v)}-f^{\prime} f^{(i v)}\right)=0
$$

and

$$
\begin{gathered}
v \frac{\partial^{4} g}{\partial y^{4}}-\frac{\partial^{3} g}{\partial t \partial y^{2}}+\frac{\alpha_{1}}{\rho} \frac{\partial^{5} g}{\partial t \partial y^{4}}+k\left(f \frac{\partial^{3} g}{\partial y^{3}}-f^{\prime \prime} \frac{\partial g}{\partial y}\right) \\
-\frac{\alpha_{1 k}}{\rho}\left(f \frac{\partial^{5} g}{\partial y^{5}}-f^{(i v)} \frac{\partial g}{\partial y}\right)=0
\end{gathered}
$$

Integrating equations (13) and (14) once with respect to $y$ using the conditions at infinity, we have

$$
v f^{\prime \prime \prime}+k\left(f f^{\prime \prime}-f^{\prime 2}\right)-\frac{\alpha_{1} k}{\rho}\left(f f^{(i v)}-2 f^{\prime} f^{\prime \prime \prime}+f^{\prime \prime 2}\right)=-k
$$

and

$$
\begin{gathered}
v \frac{\partial^{3} g}{\partial y^{3}}-\frac{\partial^{2} g}{\partial t \partial y}+\frac{\alpha_{1}}{\rho} \frac{\partial^{4} g}{\partial t \partial y^{3}}+k\left(f \frac{\partial^{2} g}{\partial y^{2}}-f^{\prime} \frac{\partial g}{\partial y}\right) \\
-\frac{\alpha_{1 k}}{\rho}\left(f \frac{\partial^{4} g}{\partial y^{4}}-f^{\prime} \frac{\partial^{3} g}{\partial y^{3}}+f^{\prime \prime} \frac{\partial^{2} g}{\partial y^{2}}-f^{\prime \prime \prime} \frac{\partial g}{\partial y}\right) \\
=0
\end{gathered}
$$

Using the non-dimensional variables

$$
\begin{aligned}
& \eta=\sqrt{\frac{k}{v}} y, \quad \tau=\Omega t, \quad f(y)=\sqrt{\frac{v}{k}} F(\eta), \\
& g(y, t)=\frac{v}{k} G(\eta, \tau), \quad \varepsilon=\frac{U}{\sqrt{v k}}, \quad \beta=\frac{\Omega}{k}
\end{aligned}
$$

in equations (15) and (16), and boundary conditions (12a) and (12b), we obtain

$$
\begin{aligned}
& F^{\prime \prime \prime}+F F^{\prime \prime}-F^{2}-W_{e}\left(F F^{(i v)}-2 F^{\prime} F^{\prime \prime \prime}+F^{\prime \prime 2}\right)=-1 \\
& F(0)=0, \quad F^{\prime}(0)=0, \quad F^{\prime}(\infty)=1
\end{aligned}
$$


and

$$
\begin{gathered}
\frac{\partial^{3} G}{\partial \eta^{3}}+F \frac{\partial^{2} G}{\partial \eta^{2}}-F^{\prime} \frac{\partial G}{\partial \eta}-W e\left(F \frac{\partial^{4} G}{\partial \eta^{4}}-F^{\prime} \frac{\partial^{3} G}{\partial \eta^{3}}\right. \\
\left.F^{\prime \prime} \frac{\partial^{2} G}{\partial \eta^{2}}-F^{\prime \prime \prime} \frac{\partial G}{\partial \eta}\right)-\beta \frac{\partial^{2} G}{\partial \tau \partial \eta}+W e \beta \frac{\partial^{4} G}{\partial \tau \partial \eta^{3}}=0 \\
G(0, \tau)=0, \quad G_{\eta}(0, \tau)=\varepsilon e^{i \tau}, G_{\eta \eta}(\infty, \tau)=\gamma
\end{gathered}
$$

where $W e=\frac{\alpha_{1} k}{\rho v}$ is the Weissenberg number.

System (18 a-b) has been solved numerically by Garg and Rajagopal [23] and Ariel [24, 25]. Following Bellman and Kalaba [26] and Garg and Rajagopal [23], the quasi-linearized form of equation (18a) is

$$
\begin{aligned}
& F_{n+1}^{(i v)}=\frac{F_{n+1}^{\prime \prime \prime}}{F_{n}}\left(2 F_{n}^{\prime}+\frac{1}{W e}\right)+F_{n+1}^{\prime \prime}\left(\frac{1}{W e}-\frac{2 F_{n}^{\prime \prime}}{F_{n}}\right) \\
& +\frac{2 F_{n+1}^{\prime}}{F_{n}}\left(F^{\prime \prime \prime}-\frac{F_{n}^{\prime}}{W e}\right) \\
& +\frac{F_{n+1}}{F_{n}^{2}}\left[F_{n}^{\prime \prime 2}-2 F_{n}^{\prime} F_{n}^{\prime \prime \prime}+\frac{F_{n}^{\prime 2}-F_{n}^{\prime \prime \prime}-1}{W e}\right]+\frac{F_{n}^{\prime \prime \prime}+2}{W e F_{n}}
\end{aligned}
$$

where the subscript $n$ and $(n+1)$ represents the $n^{\text {th }}$ and $(n+1)^{t h}$ approximation to the solution. Since the above equation is non-homogeneous, the solution at any approximation level can be written as = $F_{\text {homogeneous }}+F_{\text {particular. }}$ Further, the homogeneous solution, $F_{\text {homogeneous }}$, is a linear combination of two linearly independent solutions - namely $F_{h_{1}}$ and $F_{h_{2}}$. The details of this technique are well described by Garg and Rajagopal [23].

Using the quasi-linearization technique described by Garg and Rajagopal [23], we find that $F^{\prime \prime}(0)=$ 1.23259 when $W e=0$. This value is in good agreement with the value obtained by Takemitsu and Matunobu [16]. Numerical values of $F^{\prime \prime}(0)$ for different values of $W e$ are shown in Table 1 . These values are in good agreement with the values obtained by Garg and Rajagopal [23] and Ariel [24]. Figure 1 shows the profiles of $F^{\prime}$ for various values of $W e$. We observed that as the elasticity of the fluid increases, the velocity near the wall decreases.

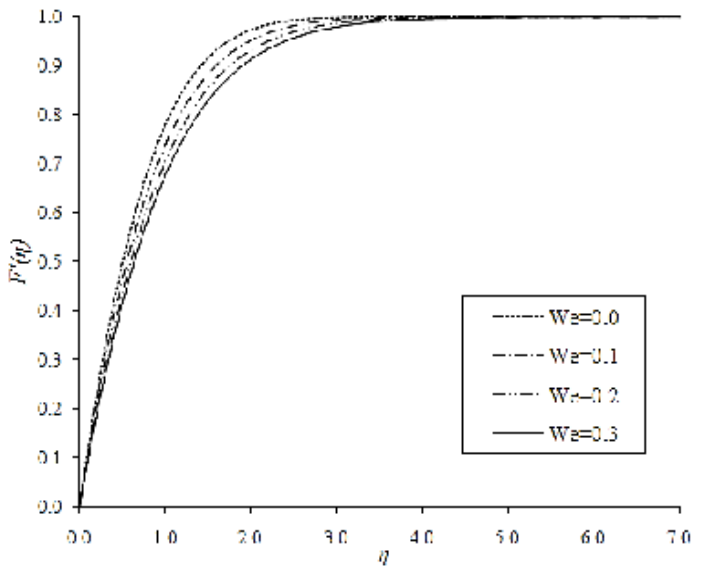

Figure 1. Variations of $F^{\prime}(\eta)$ for various values of $W e$.

Letting $G(\eta, \tau)=G_{0}(\eta)+\varepsilon G_{1}(\eta) e^{i \tau}$, then system (19) gives

$$
\begin{array}{r}
G_{0}^{\prime \prime \prime}+F G_{0}^{\prime \prime}-F^{\prime} G_{0}^{\prime} \\
-W e\left(F G_{0}^{(i v)}-F^{\prime} G_{0}^{\prime \prime \prime}+F^{\prime \prime} G_{0}^{\prime \prime}\right. \\
\left.-F^{\prime \prime \prime} G_{0}^{\prime}\right)=0
\end{array}
$$

$G_{0}(0)=0, \quad G_{0}^{\prime}(0)=0, \quad G_{0}^{\prime \prime}(\infty)=\gamma$

$$
\begin{gathered}
G_{1}^{\prime \prime \prime}+F G_{1}^{\prime \prime}-F^{\prime} G_{1}^{\prime}-W e\left(F G_{1}^{(i v)}-F^{\prime} G_{1}^{\prime \prime \prime}+F^{\prime \prime} G_{1}^{\prime \prime}\right. \\
\left.-F^{\prime \prime \prime} G_{1}^{\prime}\right)-i \beta\left(G_{1}^{\prime}-W e G_{1}^{\prime \prime \prime}\right)=0 \\
G_{1}(0)=0, \quad G_{1}^{\prime}(0)=1, \quad G_{1}^{\prime}(\infty)=0
\end{gathered}
$$

Letting $G_{0}^{\prime}(\eta)=\gamma H_{0}(\eta)$, then system (21 a-b) gives

$$
\begin{aligned}
& H_{0}^{\prime \prime}+F H_{0}^{\prime}-F^{\prime} H_{0}-W e\left(F H_{0}^{\prime \prime \prime}-F^{\prime} H_{0}^{\prime \prime}+F^{\prime \prime} H_{0}^{\prime}\right. \\
& \left.-F^{\prime \prime \prime} H_{0}\right)=0 \\
& H_{0}(0)=0, \quad H_{0}^{\prime}(\infty)=1
\end{aligned}
$$

System (23 a-b) is solved numerically using a shooting method and it is found that for $W e=0$, $H_{0}^{\prime}(0)=0.607965$. Since $G_{0}^{\prime \prime}(0)=\gamma H_{0}^{\prime}(0)$, then for $W e=0, \quad G_{0}^{\prime \prime}(0)=0.607965 \gamma$ which is in good agreement with the value obtained by Takemitsu and Matunobu [16]. Numerical values of $H_{0}^{\prime}(0)$ for different values of $W e$ are shown in Table 1 . Figure 2 depicts the profiles of $H_{0}^{\prime}$ for various values of $W e$. 


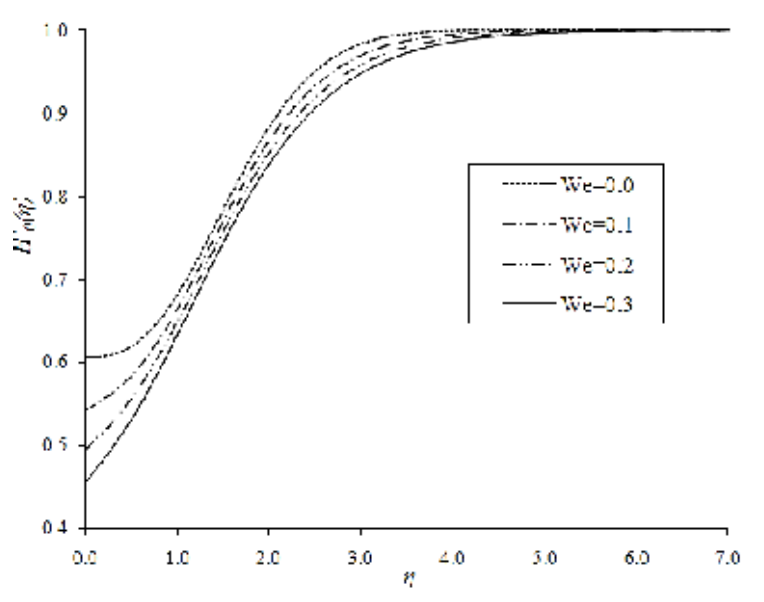

Figure 2. Variations of $H_{0}^{\prime}(\eta)$ for various values of $W e$.

Table 1. Numerical values of $F^{\prime \prime}(0), H_{0}^{\prime}(0), \phi_{0}^{\prime}(0)$, $\phi_{1}^{\prime}(0)$ and $\phi_{2}^{\prime}(0)$ for different values of $W e$.

\begin{tabular}{|c|c|c|c|c|c|}
\hline$W e$ & $F^{\prime \prime}(0)$ & $H_{0}^{\prime}(0)$ & $\phi_{0}^{\prime}(0)$ & $\phi_{1}^{\prime}(0)$ & $\phi_{2}^{\prime}(0)$ \\
\hline 0.0 & 1.23259 & 0.60777 & -0.81107 & -0.49348 & 0.09471 \\
\hline 0.1 & 1.13425 & 0.54392 & -0.76774 & 0.50612 & 0.06023 \\
\hline 0.2 & 1.05818 & 0.49546 & -0.73291 & 0.51309 & 0.02785 \\
\hline 0.3 & 0.99689 & 0.45677 & -0.70364 & 0.51685 & -0.00204 \\
\hline 0.4 & 0.94588 & 0.42465 & -0.67826 & 0.51881 & -0.02953 \\
\hline 0.5 & 0.90248 & 0.39774 & -0.65619 & 0.51922 & -0.05474 \\
\hline 1.0 & 0.75276 & 0.30691 & -0.57522 & 0.51155 & -0.15428 \\
\hline 2.0 & 0.59677 & 0.21662 & -0.48170 & 0.48461 & -0.27270 \\
\hline 5.0 & 0.41288 & 0.12046 & -0.35721 & 0.41638 & -0.39192 \\
\hline 8.0 & 0.33533 & 0.08503 & -0.29916 & 0.37114 & -0.40062 \\
\hline 10 & 0.30283 & 0.07127 & -0.27371 & 0.34885 & -0.38807 \\
\hline 20 & 0.21857 & 0.03978 & -0.20475 & 0.28684 & -0.37758 \\
\hline 50 & 0.14008 & 0.01735 & -0.13476 & 0.19505 & 0.16073 \\
\hline 100 & 0.09951 & 0.00897 & -0.09591 & 0.14291 & 0.33255 \\
\hline 200 & 0.07053 & 0.00453 & -0.06490 & 0.08305 & 1.48595 \\
\hline 500 & 0.04469 & 0.00180 & -0.02550 & -0.04243 & 3.12615 \\
\hline
\end{tabular}

Letting $\phi(\eta)=G_{1}^{\prime}(\eta)$, then system (22 a-b) becomes

$$
\begin{gathered}
\phi^{\prime \prime}+F \phi^{\prime}-F^{\prime} \phi-W e\left(F \phi^{\prime \prime \prime}-F^{\prime} \phi^{\prime \prime}+F^{\prime \prime} \phi^{\prime}-F^{\prime \prime \prime} \phi\right) \\
-i \beta\left(\phi-W e \phi^{\prime \prime}\right)=0
\end{gathered}
$$

The only parameter in equation (24a) is the frequency $\beta$. Two series solutions valid for small and large $\beta$ respectively will be obtained. For small values of the frequency $\beta$, we assume that

$$
\begin{aligned}
\phi(\eta) & =\sum_{n=0}^{\infty} \beta^{n} \phi_{n}(\eta) \\
& =\phi_{0}(\eta)+i \beta \phi_{1}(\eta)+(i \beta)^{2} \phi_{2}(\eta)+\cdots
\end{aligned}
$$

where the numerical values for $\phi_{0}^{\prime}(0), \phi_{1}^{\prime}(0)$ and $\phi_{2}^{\prime}(0)$ are given in Table 1 for different values of $W e$.

For large values of the frequency $\beta$, we let

$Y=\alpha \eta, \alpha=\sqrt{i \beta}$ and

$$
\begin{aligned}
\phi(\eta) & =\sum_{n=0}^{\infty} \alpha^{n} \phi_{n}(\eta) \\
& =\phi_{0}(\eta)+\alpha \phi_{1}(\eta)+\alpha^{2} \phi_{2}(\eta)+\cdots
\end{aligned}
$$

and it was found that

$$
\begin{aligned}
\phi^{\prime}(0)= & \phi_{0}^{\prime}(0)+\alpha \phi_{1}^{\prime}(0)+\alpha^{2} \phi_{2}^{\prime}(0)+\alpha^{3} \phi_{3}^{\prime}(0)+\cdots \\
= & -\frac{1}{\sqrt{1+m}}-\frac{(3-4 m)}{8(1+m)} F^{\prime \prime}(0) \alpha^{3}+\frac{3+4 m}{16 \sqrt{1+m}} \alpha^{4} \\
& -\frac{\left(40 m^{3}-50 m^{2}+28 m-33\right) F^{\prime \prime 2}(0)}{128(1+m) \sqrt{1+m}} \alpha^{6}+\cdots
\end{aligned}
$$

where $m \neq 1$ and the numerical values of $F^{\prime \prime}(0)$ are given in Table 1 for different values of $W e$. Figures 3-5 depict the variations of $\phi_{0}(\eta), \phi_{1}(\eta)$ and $\phi_{2}(\eta)$ for various values of $W e$.

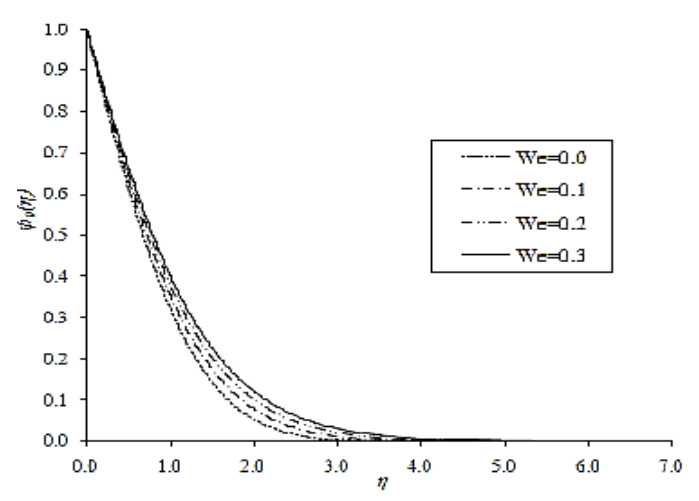

Figure 3. Variations of $\phi_{0}(\eta)$ for various values of $W e$. 


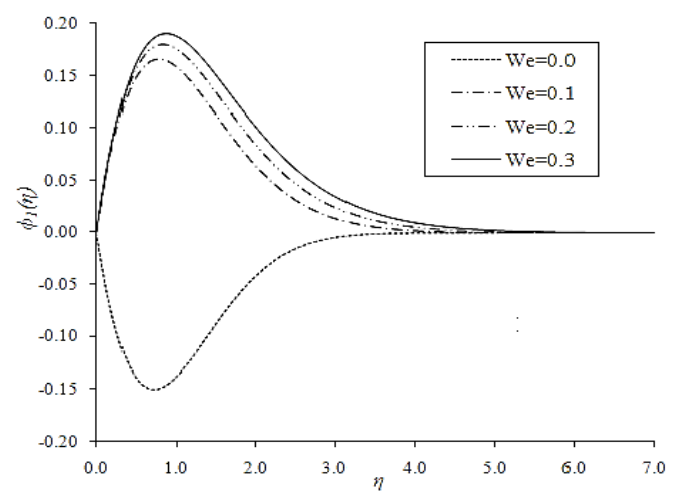

Figure 4. Variations of $\phi_{1}(\eta)$ for various values of $W e$.

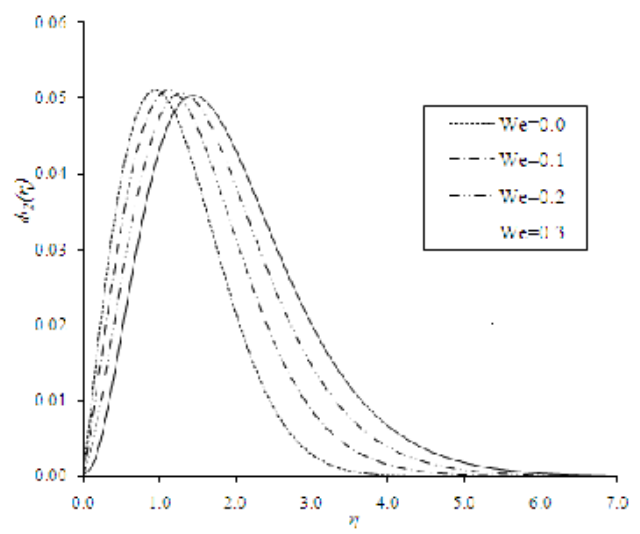

Figure 5. Variations of $\phi_{2}(\eta)$ for various values of $W e$.

\section{Solutions in the Moving Frame of Reference}

We assume that the Cartesian coordinates $(x, y)$ are moving with the plate, the $x$-axis is along the plate and the $y$-axis is normal to the plate. In this case, following Takemitsu and Matunobu [16], we assume that the streamfunction is given by

$$
\psi=k[x f(y)+h(y, t)]
$$

and the boundary conditions are given by

$$
\begin{aligned}
& f(0)=f^{\prime}(0)=0, \quad h(0, t)=0, \quad h_{y}(0, t)=0 \\
& f^{\prime}(\infty)=1, \quad h_{y}(\infty, t)=\gamma y-\frac{U}{k} e^{i \Omega t}
\end{aligned}
$$

We note that the flow is oscillating with velocity $-U \cos \Omega t$ at infinity. Using equation (28) in (9), equating different powers of $x$ to zero and integrating once with respect to $y$ using the conditions at infinity, we obtain

$$
v f^{\prime \prime \prime}+k\left(f f^{\prime \prime}-f^{\prime 2}\right)-\frac{\alpha_{1} k}{\rho}\left(f f^{(i)}-2 f^{\prime} f^{\prime \prime \prime}+f^{\prime \prime 2}\right)=-k
$$

and

$$
\begin{array}{r}
v \frac{\partial^{3} h}{\partial y^{3}}-\frac{\partial^{2} h}{\partial t \partial y}+\frac{\alpha_{1}}{\rho} \frac{\partial^{4} h}{\partial t \partial y^{3}}+k\left(f \frac{\partial^{2} h}{\partial y^{2}}-f^{\prime} \frac{\partial h}{\partial y}\right) \\
-\frac{\alpha_{1 k}}{\rho}\left(f \frac{\partial^{4} h}{\partial y^{4}}-f^{\prime} \frac{\partial^{3} h}{\partial y^{3}}+f^{\prime \prime} \frac{\partial^{2} h}{\partial y^{2}}-f^{\prime \prime \prime} \frac{\partial h}{\partial y}\right) \\
=\left(1+\frac{i \Omega}{k}\right) U e^{i \Omega t}
\end{array}
$$

Non-dimensionalizing using

$$
\begin{aligned}
& \eta=\sqrt{\frac{k}{v}} y, \quad \tau=\Omega t, \quad f(y)=\sqrt{\frac{v}{k}} F(\eta), \\
& h(y, t)=\frac{v}{k} G(\eta, \tau), \quad \beta=\frac{\Omega}{k}, \quad \varepsilon=\frac{U}{\sqrt{v k}}
\end{aligned}
$$

we obtain

$$
F^{\prime \prime \prime}+F F^{\prime \prime}-F^{\prime 2}-W_{e}\left(F F^{(i v)}-2 F^{\prime} F^{\prime \prime \prime}+F^{\prime \prime 2}\right)=-1
$$

$$
F(0)=0, \quad F^{\prime}(0)=0, \quad F^{\prime}(\infty)=1
$$

and

$$
\begin{aligned}
& \frac{\partial^{3} G}{\partial \eta^{3}}+F \frac{\partial^{2} G}{\partial \eta^{2}}-F^{\prime} \frac{\partial G}{\partial \eta}-W e\left(F \frac{\partial^{4} G}{\partial \eta^{4}}-F^{\prime} \frac{\partial^{3} G}{\partial \eta^{3}}\right. \\
& \left.F^{\prime \prime} \frac{\partial^{2} G}{\partial \eta^{2}}-F^{\prime \prime \prime} \frac{\partial G}{\partial \eta}\right)-\beta \frac{\partial^{2} G}{\partial \tau \partial \eta}+W e \beta \frac{\partial^{4} G}{\partial \tau \partial \eta^{3}}=(1+i \beta) \varepsilon e^{i \tau}
\end{aligned}
$$

$G(0, \tau)=0, \quad G_{\eta}(0, \tau)=\varepsilon e^{i \tau}, G_{\eta}(\infty, \tau)=\gamma \eta-\varepsilon e^{i \tau}$

System (33 a-b) has been solved numerically in section 3. Letting $G(\eta, \tau)=G_{0}(\eta)-\varepsilon H(\eta) e^{i \tau}$, system (34 a-b) gives

$$
\begin{gathered}
G_{0}^{\prime \prime \prime}+F G_{0}^{\prime \prime}-F^{\prime} G_{0}^{\prime}-W e\left(F G_{0}^{(i v)}-F^{\prime} G_{0}^{\prime \prime \prime}+F^{\prime \prime} G_{0}^{\prime \prime}\right. \\
\left.-F^{\prime \prime \prime} G_{0}^{\prime}\right)=0 \\
G_{0}(0)=0, \quad G_{0}^{\prime}(0)=0, \quad G_{0}^{\prime \prime}(\infty)=\gamma
\end{gathered}
$$


and

$$
\begin{aligned}
& H^{\prime \prime \prime}+F H^{\prime \prime}-F^{\prime} H^{\prime}-W e\left(F H^{(i v)}-F^{\prime} H^{\prime \prime \prime}+F^{\prime \prime} H^{\prime \prime}\right. \\
& \left.-F^{\prime \prime \prime} H^{\prime}\right)-i \beta\left(H^{\prime}-W e H^{\prime \prime \prime}\right)=-1-i \beta \\
& H(0)=0, \quad H^{\prime}(0)=1, \quad H^{\prime}(\infty)=1
\end{aligned}
$$

Numerical solutions of system (35a-b) have been obtained in section 3. It can easily be shown that the function

$H^{\prime}=\frac{F^{\prime}+i \beta G_{1}^{\prime}-i \beta}{1-i \beta}$

is a solution of system ( $36 \mathrm{a}-\mathrm{b}$ ) since it satisfies both the equation and the boundary conditions. In equation (37), the functions $F^{\prime}$ and $G_{1}^{\prime}$ have been found in section 3 .

\section{Discussion and Conclusions}

The unsteady second grade stagnation-point flow impinging obliquely on an oscillatory flat plate is studied. Numerical results for this flow are found for various values of the Weissenberg number $W e$. Figure 1 shows the variations of $F^{\prime}(\eta)$ for various values of $W e$. The effect of the Weissenberg number, $W e$, is to decrease the velocity $F^{\prime}(\eta)$ near the wall as it increases. Figure 2 depicts the variations of $H_{0}^{\prime}(\eta)$ for various values of $W e$ and shows that $H_{0}^{\prime}(\eta)$ decreases near the wall as $W e$ is increasing. The variations of $\phi_{0}(\eta)$ with various values of $W e$ are shown in Figure 3. From this figure we observed that $\phi_{0}(\eta)$ is decreasing as $W e$ is incresing. Figure 4 shows the variations of $\phi_{1}(\eta)$ for various values of $W e$ and Figure 5 depicts the variations of $\phi_{2}(\eta)$ for various values of $W e$. From Table $1, F^{\prime \prime}(0)$ is decreasing as $W e$ is increasing.

\section{References}

[1] K. Hiemenz, "Die Grenzschicht an einem in den gleichformigen Flussigkeitsstrom eingetauchten geraden Kreiszylinder," Dingler's Polytech., vol. 326, pp. 321, 1911.

[2] J. T. Stuart, "The viscous flow near a stagnation point when the external flow has uniform vorticity," J. Aerospace Sci., vol. 26, pp. 124, 1959.

[3] K. J. Tamada, "Two-dimensional stagnation point flow impinging obliquely on a plane wall," J. Phys. Soc. Japan, vol. 46, pp. 310, 1979.

[4] J. M. Dorrepaal, "An Exact solution of the NavierStokes equation which describes non-orthogonal stagnation-point flow in two dimensions," J. Fluid Mech., vol. 163, pp. 141, 1986.

[5] B. W. Beard and K. Walters, "Elastico-viscous boundary layer flows," in Proc. Camb. Phil. Soc., 1964.

[6] J. M. Dorrepaal, O. P. Chandna and F. Labropulu, "The flow of a visco-elastic fluid near a point of reattachment," ZAMP, vol. 43, pp. 708, 1992.

[7] F. Labropulu, J. M. Dorrepaal and O. P. Chandna, "Viscoelastic fluid flow impinging on a wall with suction or blowing," Mech. Res. Comm., vol. 20, no. 2, pp. 143, 1993.

[8] N. Rott, "Unsteady viscous flow in the vicinity of a stagnation point," Quart. of Appl. Math, vol. 13, pp. 444, 1956.

[9] M. B. Glauert, "The laminar boundary layer on oscillating plates and cylinders," J. Fluid Mech., vol. 1, pp. 97, 1956.

[10] A. C. Srivastava, "Unsteady flow of a second-order fluid near a stagnation point," J. Fluid Mech., vol. 24, no. Part 1, pp. 33, 1966.

[11] C. H. Yih, Fluid Mechanics, New York: West River Press, 1977.

[12] F. Labropulu, X. Xu and M. Chinichian, "Unsteady Stagnation Point Flow of a non-Newtonian SecondGrade Fluid," Int. J. Math. \& Math. Sci., vol. 2003, no. 50, pp. 3797-3807, 2003.

[13] Y. Matunobu, "Structure of pulsatile Hiemenz flow and temporal variation of wall shear stress near the stagnation point. I," J. Phys. Soc. Japan, vol. 42, pp. 2041, 1977.

[14] Y. Matunobu, "Structure of pulsatile Hiemenz flow and temporal variation of wall shear stress near the stagnation point. II," J. Phys. Soc. Japan, vol. 43, pp. 326, 1977.

[15] M. Kawaguti and K. Hamano, "Two-dimensional model of pulsatile flow through constricted artery," in Xth Intern. Congr. Angiology, 1976.

[16] N. Takemitsu and Y. Matunobu, "Unsteady stagnation-point flow impinging oqliquely on an oscillating flat plate," J. Phys. Soc. Japan, vol. 47, no. 4, pp. 1347, 1979.

[17] Y. Matunobu and M. Arakawa, "Model experiment on the post-stenotic dilatation in blood vessels," Biorhelogy, vol. 11, pp. 427, 1974.

[18] B. Gessner, "Hemodynamic theories of atherogenesis," Circulation Research, vol. 33, pp. 259, 1973. 
[19] R. S. Rivlin and J. L. Ericksen, "Stress-deformation relations for isotropic materials," J. Rat. Mech. Anal., vol. 4, pp. 323, 1955.

[20] J. E. Dunn and R. L. Fosdick, "Thermodynamics, stability and boundedness of fluids of complexity 2 and fluids of second grade," Arch. Rational Mech. Anal., vol. 56, pp. 191, 1974.

[21] J. E. Dunn and K. R. Rajagopal, "Fluids of differential type:critical review and thermodynamic analysis," Int. J. Eng. Sci., vol. 33, pp. 689, 1995.

[22] C. Truesdell, "The Mechanical foundations of elasticity and fluid dynamics," Journal of Rational Mechanics and Analysis, vol. 1, pp. 125, 1952.

[23] V. K. Garg and K. R. Rajagopal, "Stagnation point flow of a non-Newtonain fluid," Mech. Res. Comm., vol. 17, no. 6, pp. 415, 1990.

[24] P. D. Ariel, "A hybrid method for computing the flow of viscoelastic fluids," Int. J. Num. Meth. in Fluids, vol. 14, pp. 323, 1992.

[25] P. D. Ariel, "On extra boundary condition in the stagnation point flow of a second grade fluid," Int. J. Engng. Sci, vol. 40, pp. 145, 2002.

[26] R. E. Bellman and R. E. Kalaba, in Quasilinearization and Non-Linear Boundary Value Problems, New York, Elsevier, 1965. 